\title{
Genital Infection as a First Sign of Acute Myeloid Leukemia
}

\author{
Naoki Oiso ${ }^{a}$ Shinya Rai ${ }^{b}$ Shigeru Kawara ${ }^{a}$ \\ Yoichi Tatsumi $^{\text {b }}$ Akira Kawada ${ }^{a}$
}

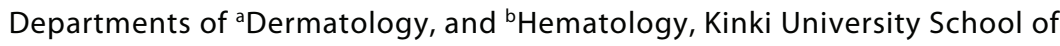

Medicine, Osaka-Sayama, Japan

\section{Key Words}

Bacterial infection · Fournier's gangrene - Necrotizing fasciitis - Hematologic malignancy · Acute myeloid leukemia

\begin{abstract}
Fournier's gangrene is a life-threatening disorder caused by aerobic and anaerobic bacterial infection. We report a case of genital infection as the initial warning sign of acute myeloid leukemia. We were able to prevent progression to Fournier's gangrene in our patient by immediate intensive therapy with incision, blood transfusions and intravenous administration of antibiotics. This case suggests that hematologists and dermatologists should keep in mind that genital infection can be a first sign of hematologic malignancy.
\end{abstract}

\section{Introduction}

Fournier's gangrene (FG) is a life-threatening disorder caused by synergistic aerobic and anaerobic organisms [1]. The infection of the perineum, scrotum, and/or penis spreads along fascial planes, leading to soft-tissue necrosis [1]. The mortality rate for FG remains high despite antibiotics and aggressive debridement [1]. The initial signs of FG are fever, pain, swelling, and blistering in the genital area [2]. Here, we describe a case of genital infection as the first sign of acute myeloid leukemia (AML).

\section{Case Report}

A 51-year-old male presented with fever and a painful, edematous erythema on the scrotum and penis (fig. 1a, b). The eruption had developed after the patient had ridden a bicycle 7 days earlier. Laboratory blood examination results were as follows: white blood cells 7,800/ $\mu$ with neutrophil $12.0 \%$; lymphocytes $4.7 \%$; monocytes $0.3 \%$; myeloblasts $83.0 \%$; red blood cells $1.97 \times 10^{6} / \mu$ l; platelets $26.4 \times$ $10^{4} / \mu \mathrm{l}$, and C-reactive protein $4.621 \mathrm{mg} / \mathrm{dl}$. The smear from the bone marrow indicated the presence of a massive myeloblast. The cells expressed CD7, CD11, CD13, CD14, and CD34. An incision was made in the scrotum. Corynebacterium spp. was isolated from the genital region. The patient was immediately 
hospitalized and intensively treated with blood transfusions and the antibiotics cefpirome sulfate $4 \mathrm{~g}$ and clindamycin 2,400 mg per day for 5 days and cilastatin sodium $2 \mathrm{~g}$ and clindamycin 2,400 $\mathrm{mg}$ per day for 9 days. The sign of infection diminished two weeks later. Treatment for AML was then initiated using cytarabine, aclarubicin, and granulocyte-colony stimulating factor.

\section{Discussion}

FG was mainly caused by trauma and urinary tract infection [3]. In our case, we suspect that the trauma was due to the cycling activity and that the infection was worsened by leukocytopenia due to AML. Corynebacterium spp. was cultured from the scrotum. We do not think that Corynebacterium spp. was the actual pathologic bacteria because Corynebacterium spp. is part of the normal cutaneous flora in the genital area. We believe that our patient showed scrotum infection by unidentified bacteria and that intensive medication prevented progression to ulceration and the more advanced stage of necrosis as a sign of FG.

Few cases of FG have been described as a first sign of hematologic malignancies [4, 5], although cases have been reported as a complication during treatment (table 1) [2, 6-11]. Martinelli et al. [2] reported a fetal case having progressive involvement of the abdominal wall resulting in death from leukemia. They indicated that early diagnosis of the disorder and appropriate initiation of an accurate therapy can prevent progression of the acute necrotizing infection [2]. In the reported cases of FG associated with a hematologic malignancy, the edema and swelling were the initial signs $[2,5,7,9,10]$. The immediate start of the treatment might have prevented the present patient from being affected by FG.

Hematologists and dermatologists should keep in mind that genital infection and its advanced stage of FG can be an initial sign of hematologic malignancy.

Table 1. Fournier's gangrene or genital infection associated with hematologic malignancies: a summary of the reported cases

\begin{tabular}{|c|c|c|c|c|c|c|}
\hline Case & $\begin{array}{l}\text { Age/ } \\
\text { sex }\end{array}$ & $\begin{array}{l}\text { Onset of } \\
\text { Fournier's } \\
\text { gangrene }^{\mathrm{a}}\end{array}$ & $\begin{array}{l}\text { Hematologic } \\
\text { malignancy }\end{array}$ & $\begin{array}{l}\text { Clinical feature of the } \\
\text { onset }\end{array}$ & $\begin{array}{l}\text { Clinical feature of the severest } \\
\text { situation }\end{array}$ & $\begin{array}{l}\text { Phlogogenous } \\
\text { bacteria }\end{array}$ \\
\hline $\begin{array}{l}\text { Naithani } \\
\text { et al., } \\
2008 \text { [6] }\end{array}$ & $17 / \mathrm{M}$ & $\begin{array}{l}\text { after } \\
\text { diagnosis }\end{array}$ & $\begin{array}{l}\text { acute promyelocytic } \\
\text { leukemia }\end{array}$ & $\begin{array}{l}\text { painful vesicular lesions } \\
\text { in scrotum }\end{array}$ & ulcers & $\begin{array}{l}\text { Staphylococcus } \\
\text { aureus, Escherichia } \\
\text { coli }\end{array}$ \\
\hline $\begin{array}{l}\text { Mantadakis } \\
\text { et al., } \\
2007 \text { [7] }\end{array}$ & $21 / \mathrm{M}$ & $\begin{array}{l}\text { after } \\
\text { diagnosis }\end{array}$ & $\begin{array}{l}\text { acute lymphoblastic } \\
\text { leukemia }\end{array}$ & scrotal edema & a small necrotic scrotal eschar & $\begin{array}{l}\text { Pseudomonas } \\
\text { aeruginosa, } \\
\text { Staphylococcus } \\
\text { epidermidis }\end{array}$ \\
\hline $\begin{array}{l}\text { Fukuno } \\
\text { et al., } \\
2003 \text { [8] }\end{array}$ & $43 / \mathrm{M}$ & $\begin{array}{l}\text { after } \\
\text { diagnosis }\end{array}$ & $\begin{array}{l}\text { acute promyelocytic } \\
\text { leukemia }\end{array}$ & $\begin{array}{l}\text { an ulcer of } 0.5 \mathrm{~cm} \text { in } \\
\text { diameter on the left side } \\
\text { of the scrotum }\end{array}$ & $\begin{array}{l}\text { swelling that was improved by a } \\
\text { surgical incision }\end{array}$ & no description \\
\hline $\begin{array}{l}\text { Bakshi } \\
\text { et al., } \\
2003 \text { [9] } \\
\text { 1st case }\end{array}$ & $6 / \mathrm{M}$ & $\begin{array}{l}\text { after } \\
\text { diagnosis }\end{array}$ & $\begin{array}{l}\text { acute myeloid } \\
\text { leukemia }\end{array}$ & edema over the prepuce & a necrotic ulcer & $\begin{array}{l}\text { Pseudomonas } \\
\text { aeruginosa }\end{array}$ \\
\hline $\begin{array}{l}\text { Bakshi } \\
\text { et al., } \\
\text { 2003 [9] } \\
\text { 2nd case }\end{array}$ & $10 / \mathrm{M}$ & $\begin{array}{l}\text { after } \\
\text { diagnosis }\end{array}$ & $\begin{array}{l}\text { acute lymphoblastic } \\
\text { leukemia }\end{array}$ & $\begin{array}{l}\text { pain and swelling in the } \\
\text { prepuce }\end{array}$ & abscess & $\begin{array}{l}\text { Pseudomonas } \\
\text { aeruginosa }\end{array}$ \\
\hline $\begin{array}{l}\text { Bakshi } \\
\text { et al., } \\
2003 \text { [9] } \\
\text { 3rd case }\end{array}$ & $9 / \mathrm{M}$ & $\begin{array}{l}\text { after } \\
\text { diagnosis }\end{array}$ & $\begin{array}{l}\text { non-Hodgkin } \\
\text { lymphoma }\end{array}$ & $\begin{array}{l}\text { severe pain during } \\
\text { micturition, erythema, } \\
\text { and tenderness in the } \\
\text { penile region }\end{array}$ & $\begin{array}{l}\text { gangrenous changes on the } \\
\text { prepuce and glans }\end{array}$ & no description \\
\hline
\end{tabular}




\begin{tabular}{|c|c|c|c|c|c|c|}
\hline $\begin{array}{l}\text { Yoshda } \\
\text { et al., } \\
2002[10]\end{array}$ & $16 / \mathrm{M}$ & $\begin{array}{l}\text { after } \\
\text { diagnosis }\end{array}$ & $\begin{array}{l}\text { acute myelogenous } \\
\text { leukemia }\end{array}$ & $\begin{array}{l}\text { penile swelling with } \\
\text { miction pain }\end{array}$ & $\begin{array}{l}\text { gangrene in the regions of the } \\
\text { scrotum, penis, thighs, and lower } \\
\text { abdomen }\end{array}$ & $\begin{array}{l}\text { Pseudomonas } \\
\text { aeruginosa }\end{array}$ \\
\hline $\begin{array}{l}\text { Islamoglu } \\
\text { et al., } \\
2001[5]\end{array}$ & $33 / \mathrm{M}$ & $\begin{array}{l}\text { before } \\
\text { diagnosis }\end{array}$ & $\begin{array}{l}\text { acute } \\
\text { myelomonocytic } \\
\text { leukemia }\end{array}$ & scrotum edema & $\begin{array}{l}\text { complete scrotal necrosis, } \\
\text { complete penile shaft necrosis, } \\
\text { and a left anal ulcer that } \\
\text { extended to the left gluteal area }\end{array}$ & Bacteroides fragilis \\
\hline $\begin{array}{l}\text { Martinelli } \\
\text { et al., } \\
1998 \text { [2] } \\
1 \text { st case }\end{array}$ & $41 / \mathrm{M}$ & $\begin{array}{l}\text { after } \\
\text { diagnosis }\end{array}$ & $\begin{array}{l}\text { acute non- } \\
\text { lymphocytic } \\
\text { leukemia }\end{array}$ & $\begin{array}{l}\text { genital erythema, pain, } \\
\text { swelling and crepitation }\end{array}$ & blistering and ulceration & $\begin{array}{l}\text { Pseudomonas } \\
\text { aeruginosa }\end{array}$ \\
\hline $\begin{array}{l}\text { Martinelli } \\
\text { et al., } \\
1998 \text { [2] } \\
\text { 2nd case }\end{array}$ & $26 / \mathrm{F}$ & $\begin{array}{l}\text { after } \\
\text { diagnosis }\end{array}$ & $\begin{array}{l}\text { acute non- } \\
\text { lymphocytic } \\
\text { leukemia }\end{array}$ & $\begin{array}{l}\text { redness and swelling of } \\
\text { the right labium } \\
\text { majorum }\end{array}$ & ulceration & $\begin{array}{l}\text { Pseudomonas } \\
\text { aeruginosa }\end{array}$ \\
\hline $\begin{array}{l}\text { Martinelli } \\
\text { et al., } \\
1998 \text { [2] } \\
\text { 3rd case }\end{array}$ & $26 / \mathrm{F}$ & $\begin{array}{l}\text { after } \\
\text { diagnosis }\end{array}$ & $\begin{array}{l}\text { acute non- } \\
\text { lymphocytic } \\
\text { leukemia }\end{array}$ & $\begin{array}{l}\text { pain, edema, erythema } \\
\text { and swelling of the } \\
\text { perineal area }\end{array}$ & a necrotic ulcer & $\begin{array}{l}\text { Pseudomonas } \\
\text { aeruginosa }\end{array}$ \\
\hline $\begin{array}{l}\text { Faber } \\
\text { et al., } \\
1998[4]\end{array}$ & $50 / \mathrm{M}$ & $\begin{array}{l}\text { before } \\
\text { diagnosis }\end{array}$ & $\begin{array}{l}\text { acute myelogenous } \\
\text { leukemia }\end{array}$ & $\begin{array}{l}\text { progressive perianal } \\
\text { pain }\end{array}$ & $\begin{array}{l}\text { a diffusely infiltrated anal region } \\
\text { and bluish scrotum }\end{array}$ & Escherichia coli \\
\hline $\begin{array}{l}\text { Levy } \\
\text { et al., } \\
1998[11]\end{array}$ & $44 / \mathrm{M}$ & $\begin{array}{l}\text { after } \\
\text { diagnosis }\end{array}$ & $\begin{array}{l}\text { acute promyelocytic } \\
\text { leukemia }\end{array}$ & $\begin{array}{l}\text { small indurated lesion } \\
\text { of the right scrotum }\end{array}$ & a painful necrotic area $4 \times 5 \mathrm{~cm}$ & $\begin{array}{l}\text { Streptococcus faecalis, } \\
\text { Staphylococcus } \\
\text { coagulase negative }\end{array}$ \\
\hline
\end{tabular}

a The onset of Fournier's gangrene or genital infection before or after the diagnosis of a hematologic malignancy.

Fig. 1. a A painful and edematous erythema was present on the scrotum and associated with fever. b An edema was observed on the penis.

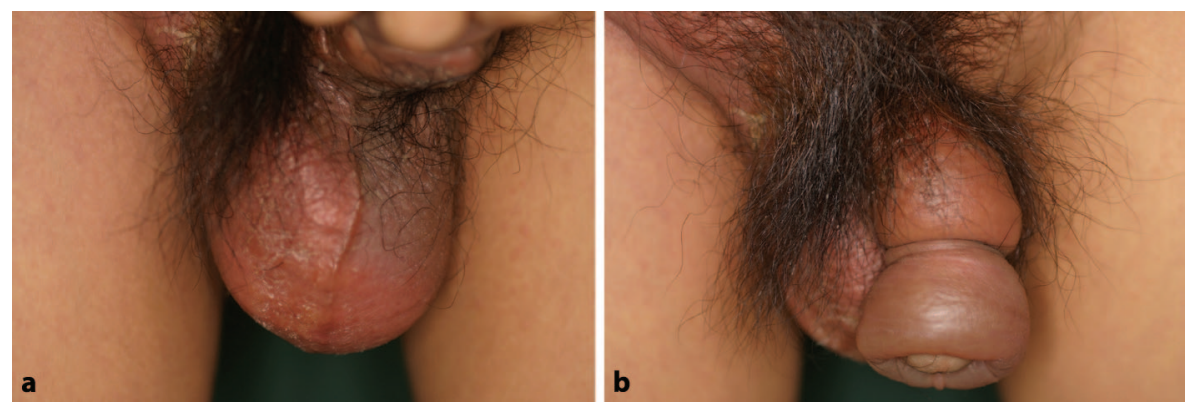




\section{References}

1 Ghnnam WM: Fournier's gangrene in Mansoura Egypt: a review of 74 cases. J Postgrad Med 2008;54:106-109.

2 Martinelli G, Alessandrino EP, Bernasconi P, Caldera D, Colombo A, Malcovati L, Gaviglio MR, Vignoli GP, Borroni G, Bernasconi C: Fournier's gangrene: a clinical presentation of necrotizing fasciitis after bone marrow transplantation. Bone Marrow Transplant 1998;22:1023-1026.

3 Bhatnagar AM, Mohite PN, Suthar M: Fournier's gangrene: a review of 110 cases for aetiology, predisposing conditions, microorganisms, and modalities for coverage of necrosed scrotum with bare testes. N Z Med J 2008;121:46-56.

4 Faber HJ, Girbes AR, Daenen S: Fournier's gangrene as first presentation of promyelocytic leukemia. Leuk Res 1998;22:473-476.

-5 Islamoglu K, Serdaroglu I, Ozgentas E: Co-occurrence of Fournier's gangrene and pancytopenia may be the first sign of acute myelomonocytic leukemia. Ann Plast Surg 2001;47:352-353.

-6 Naithani R, Kumar R, Mahapatra M: Fournier's gangrene and scrotal ulcerations during all-trans-retinoic acid therapy for acute promyelocytic leukemia. Pediatr Blood Cancer 2008;51:303-304.

7 Mantadakis E, Pontikoglou C, Papadaki HA, Aggelidakis G, Samonis G: Fatal Fournier's gangrene in a young adult with acute lymphoblastic leukemia. Pediatr Blood Cancer 2007;49:862-864.

8 Fukuno K, Tsurumi H, Goto H, Oyama M, Tanabashi S, Moriwaki H: Genital ulcers during treatment with ALL-trans retinoic acid for acute promyelocytic leukemia. Leuk Lymphoma 2003;44:2009-2013.

-9 Bakshi C, Banavali S, Lokeshwar N, Prasad R, Advani S: Clustering of Fournier (male genital) gangrene cases in a pediatric cancer ward. Med Pediatr Oncol 2003;41:472-474.

10 Yoshida C, Kojima K, Shinagawa K, Hashimoto D, Asakura S, Takata S, Tanimoto M: Fournier's gangrene after unrelated cord blood stem cell transplantation. Ann Hematol 2002;81:538-539.

11 Levy V, Jaffarbey J, Aouad K, Zittoun R: Fournier's gangrene during induction treatment of acute promyelocytic leukemia, a case report. Ann Hematol 1998;76:91-92. 